\title{
NMDA Receptor Hypofunction in the Aging-Associated Malfunction of Peripheral Tissue
}

\author{
Angélica Rivera-Villaseñor ${ }^{1}$, Frida Higinio-Rodríguez'1 , Laura Nava-Gómez 1,2, \\ Bárbara Vázquez-Prieto ${ }^{1}$, Isnarhazni Calero-Vargas ${ }^{1}$, Rafael Olivares-Moreno ${ }^{3}$ and \\ Mónica López-Hidalgo ${ }^{1 *}$ \\ ${ }^{1}$ Escuela Nacional de Estudios Superiores, Unidad Juriquilla, Universidad Nacional Autónoma de México, Mexico City, \\ Mexico, ${ }^{2}$ Facultad de Medicina, Universidad Autónoma de Querétaro, Querétaro, Mexico, ${ }^{3}$ Instituto de Neurobiología, \\ Universidad Nacional Autónoma de México, Juriquilla, Mexico
}

Glutamatergic transmission through NMDA receptors (NMDARs) is important for the function of peripheral tissues. In the bone, NMDARs and its co-agonist, D-serine participate in all the phases of the remodeling. In the vasculature, NMDARs exerts a tonic vasodilation decreasing blood perfusion in the corpus cavernosum and the filtration rate in the renal glomerulus. NMDARs are relevant for the skin turnover regulating the proliferation and differentiation of keratinocytes and the formation of the cornified envelope (CE). The interference with NMDAR function in the skin leads to a slow turnover and repair. As occurs with the brain and cognitive functions, the manifestations of a hypofunction of NMDARs resembles those observed during aging. This raises the question if the deterioration of the glomerular vasculature, the bone remodeling and the skin turnover associated with age could be related with a hypofunction of NMDARs. Furthermore, the interference of D-serine and the effects of its supplementation on these tissues, suggest that a decrease of D-serine could account for this hypofunction pointing out D-serine as a potential therapeutic target to reduce or even prevent the detriment of the peripheral tissue associated with aging.

Keywords: hypofunction of NMDA receptor, aging, skin turnover, bone remodelling, glomerulus, D-serine

\section{NMDARs PARTICIPATION IN THE REMODELING PROCESS OF THE BONE}

Bone is a connective tissue with structural and support functions providing a hard but lightweight frame to anchor the muscles. It protects the nervous system, various internal organs, and plays important roles in metabolic and homeostatic functions (Al-Suhaimi and Al-Jafary, 2020). Healthy bone tissue is constantly remodeling and requires the communication between boneresorbing (osteoclast) and bone-forming cells (osteoblast) to maintain minerals homeostasis. This process is divided into four sequential phases: activation, resorption, reversal, and formation (Langdahl et al., 2016).

Glutamate release by chondrocytes, osteoclast and osteoblast plays an important role regulating bone remodeling through the activation of glutamate transporters, metabotropic and ionotropic receptors expressed in bone cells (Genever and Skerry, 2001; Hinoi et al., 2002; Morimoto et al., 2006). In particular, in the activation phase, NMDARs expressed in chondrocytes regulate the transduction of mechanical forces in the cartilage that can trigger the beginning of the cycle (Ramage et al., 2008). Osteocytes release paracrine factors such as Receptor Activator of Nuclear 
Factor $\kappa-\mathrm{B}$ Ligand (RANKL) and osteoprotegerin (OPG) to recruit and activate circulating osteoclast to prepare the bone surface with lining cells. In the resorption phase, osteoclastogenesis requires the activation of NMDAR expressed by osteoclast precursors through the induction of the nuclear translocation of NF-kappa B (Merle et al., 2003; Wu et al., 2016). Then, osteoclasts migrate to the target zone forming a sealing zone limiting the resorption of the remodeling (Nakamura et al., 1996; Wu et al., 2017). They create an acidic microenvironment to demineralize and degrade the bone matrix to remove bone products through transcytosis before entering into apoptosis (Touaitahuata et al., 2014). Before the new bone is generated, preosteoblasts differentiate into mature osteoblasts in the reversal phase, a process that is also dependent on NMDARs through the regulation of alkaline phosphatase activity (Dobson and Skerry, 2000). In this phase, lining cells envelop and digest collagen fibrils derivate from cavities made by osteoclast (Everts et al., 2002) to prepare the bone surface depositing collagen into the bone matrix (Abdelgawad et al., 2016). Finally, during the formation phase, osteoblasts deposit collagen type I, alkaline phosphatase, and osteocalcin in a NMDAR activity dependent manner (Hinoi et al., 2002; Brown et al., 2013). Once the cellular bone matrix is formed, osteoblast can either dedifferentiate to osteocytes (Sawa et al., 2019), convert into bone progenitors (Knopf et al., 2011), or initiated apoptosis (Jilka et al., 1998).

The NR1 subunits are expressed in the bone cells and contain a site that binds glycine or D-serine that is necessary for NMDAR activation (Patton et al., 1998; Itzstein et al., 2001). In particular, $\mathrm{D}$-serine is synthesized from L-serine by the action of serine racemase (SR) (Wolosker et al., 1999). Its activity is optimum at $\mathrm{pH} 8-9$, being 6 times higher than in physiological $\mathrm{pH}$ (7.4) (Wolosker et al., 1999). The degradation of D-serine is carried out mainly by the enzyme D-amino acid oxidase (DAAO), a flavoprotein that oxidates $\mathrm{D}$-serine through the reduction of the cofactor FAD that results in the corresponding alpha-keto acid (hydroxypyruvate), hydrogen peroxide and ammonia (Sacchi et al., 2013), however, it has also been reported that SR can catalyzes the degradation of $\mathrm{D}$-serine through the $\alpha, \beta$-elimination of water (Foltyn et al., 2005; Figure 1). In the bone, SR are expressed in chondrocytes (Takarada et al., 2009) and osteoblast, however, no expression of DAAO was found in culture osteoblast and osteoclast (Takarada et al., 2012) suggesting that D-serine catabolism could occur in the kidney. Besides D-serine effects regulating NMDAR activity, it can act through amino acid transporters, $\mathrm{ATB}^{+}$and ASCT2, to promote the differentiation and maturation of osteoclasts. Because D-serine is released by osteoblast and does not change osteoblastogenesis, a paracrine effect of osteoblast-derived D-serine onto neighboring osteoclast has been proposed (Takarada et al., 2012) suggesting that in physiological conditions, $\mathrm{D}$-serine could be inhibiting the boneresorbing process (Figure 1).

In physiological conditions, NMDARs activation and D-serine are necessary to maintain a balance between bone-formation and absorption processes, and could account for alterations as occurs with aging. Aged bone is characterized by an unbalance toward bone-resorbing process that leads to a progressive decline in the bone mineral density (BMD) leading to osteoporosis.
During aging, there is a trend to reduce physical activity that contributes to the decrease of a bone-formation process (Langdahl et al., 2016). In a rat model of disuse osteoporosis induced by mechanical unloading, Ho et al. (2005) reported a decrease of $\mathrm{BMD}$, trabecular volume and osteogenic gene expression (collagen type 1 and cbfa1/Runx2). These changes were accompanied with a decrease in NR1 and NR2 subunits of NMDARs in osteoblasts but not in osteoclasts. Furthermore, an antagonist of NMDARs mimicked the down-regulation of osteogenic genes observed in disused bones. This evidence suggests that in this osteoporosis, NMDAR activity could be responsible for the decrease in BMD due an interference with osteoblast function. Furthermore, it suggests that mechanical stress due to physical activity is necessary for NMDAR expression in osteoblast.

In parallel, the decrease in BMD induced in an ovariectomized mice (a model of postmenopausal osteoporosis), glutamate administration (i.p.) reduced the BMD lost in compare with ovariectomized control mice (Hinoi et al., 2007). The authors proposed that glutamate would promote osteoblastogenesis mediated by Runx2 through NMDARs activation and may suppress osteoclastogenesis mediated by NF-kB through the cystine/Glu antiporter located in osteoclasts (Yoneda, 2017). This result shows the relevance of NMDARs activity in osteoporosis and suggest that in aged subjects, the decrease in BMD induced by a decrease in physical activity or sexual hormones in females would lead to a hypofunction of NMDARs in osteoblast and hence a reduction in mature osteoblast. This would reduce the synthesis of D-serine by osteoblast and would reduce the inhibition of $\mathrm{D}$-serine on osteoclastogenesis favoring resorption process and hence osteoporosis (Figure 1) (Steinert and Marekov, 1999; Candi et al., 2001; Kalinin et al., 2002).

\section{NMDARs AS A REGULATOR OF PERIPHERAL VASCULATURE}

The vascular system is made up of vessels whose main function is to supply nutrients, oxygen, and to remove waste products (Tennant and McGeachie, 1990; Eble and Niland, 2009). The vascular endothelium is a dynamic element that responds to vasoconstrictive and vasodilatory factors (Duffy et al., 1998) like nitric oxide (NO) (Michel and Vanhoutte., 2010; Gradinaru et al., 2015). In particular, NO is synthetized by the endothelial nitric oxide synthase (eNOS) and depends on the association of $\mathrm{Ca}^{2+}$ calmodulin to activate the eNOS and convert L-arginine in NO. This gas diffuses across the endothelium to the smooth muscle where it increases the levels of cyclic guanosine monophosphate (cGMP) and reduces the smooth muscle tension causing vasodilation (Sandoo et al., 2010). In the central nervous system, a major regulator of eNOS activity are NMDARs (LeMaistre et al., 2012; Lu et al., 2019). In the brain endothelial cells, NMDAR activation induces vasodilation through the increase in $\mathrm{NO}$ production in a $\mathrm{D}$-serine concentration-dependent manner (LeMaistre et al., 2012; Lu et al., 2019).

In addition to the above-mentioned functions of the vasculature, there are arrangements of vessels that account 


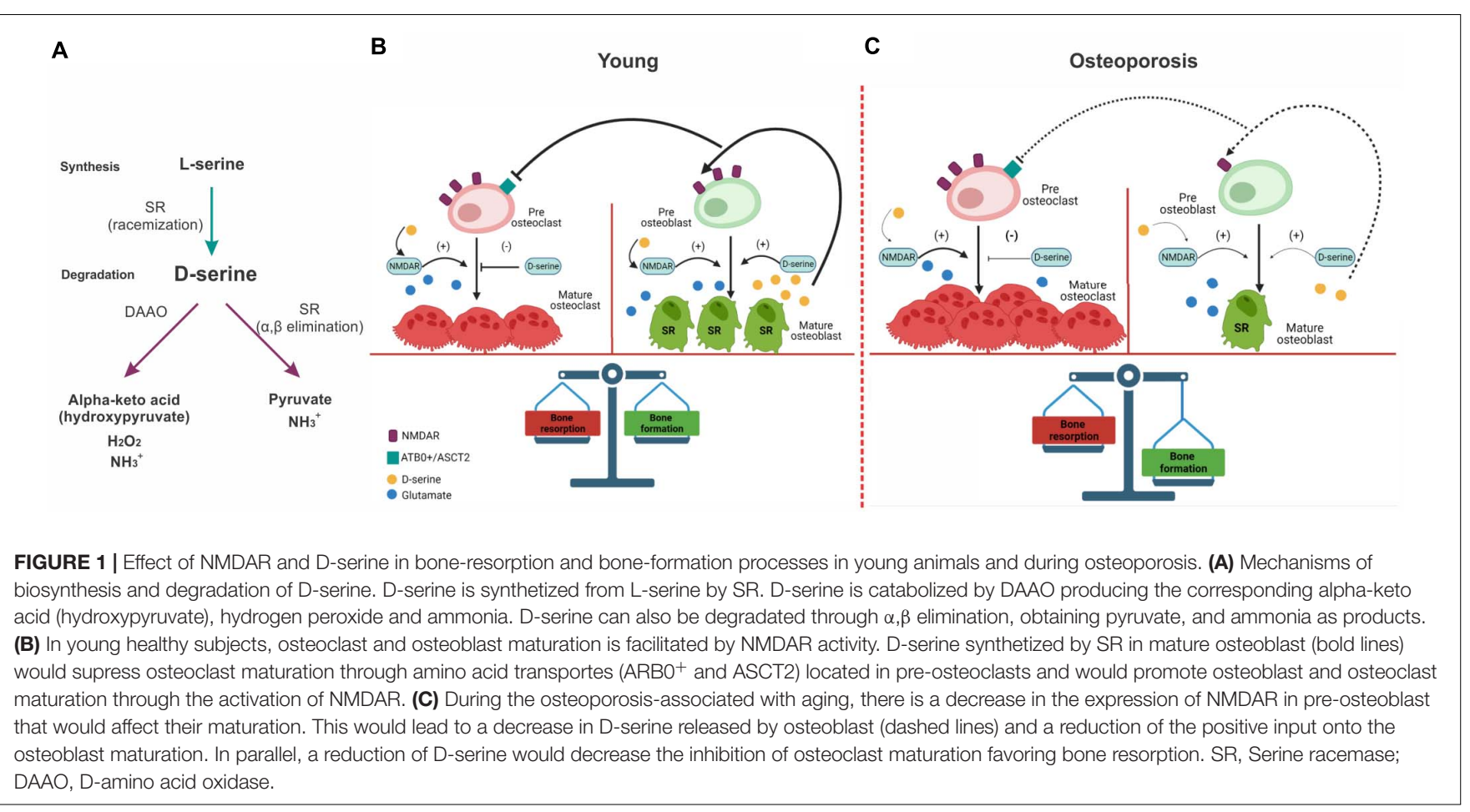

for specific functions, for example, in the glomerulus of the kidney, blood vessels determine the filtration rate of the blood to produce the urine. Here, glutamate exocytosis from mature podocytes can modulate in an autocrine/paracrine manner the activity of NMDAR located in the podocytes (Giardino et al., 2009) to regulate vascular tone. This was first revealed by Deng and colleagues, in 2002 where they showed the expression of NR1 subunit of NMDAR (Deng et al., 2002). The blockade of this site with DCKA or blocking the NMDARs with MK801, cause a significant vasoconstriction, a decrease in the renal blood flow and a reduction of the glomerular filtrate rate (Blantz et al., 2002). This suggests that tonic NMDAR activation vasodilates glomerular capillaries increasing renal blood flow (Deng and Thomson, 2009). Although SR has been reported in the kidney convoluted tubules (Xia et al., 2004), the glomeruli does not expressed SR (Xia et al., 2004) suggesting that plasmatic D-serine could act on glomerular NR1 subunit. DAAO mRNA and the protein is abundant in proximal tubules of the kidney (Koibuchi et al., 1995; Sasabe et al., 2014), however, no enzyme activity was found in glomeruli (Chan et al., 1979). This suggest that NMDAR in the glomeruli would be under the regulation of the levels of D-serine in the blood.

In the glomerulus, the vascular side is covered by endothelial cells separated from the urinary space by the basement membrane that is enfolded by podocytes (Wiggins, 2012) and whose functions in the filtration barrier depend on NMDAR activity. Here, the interference of podocytes glutamate exocytosis in $\mathrm{Rab} 3 \mathrm{~A} / \mathrm{KO}$ mice, induced a disorganization in the structure of podocytes foot process and macroalbuminuria. Furthermore, MK-801 increases albumin permeability, and remodel podocyte cytoskeleton decreasing actin and myosin/IIA as well as nephrin.
These structural changes were accompanied with proteinuria (Giardino et al., 2009). In immortalized podocytes, NMDAmediated currents were strongly potentiated by $\mathrm{D}$-serine, but not by glycine suggesting that $\mathrm{D}$-serine is the endogenous co-agonist of NMDAR in the glomerulus (Anderson et al., 2011).

The effect of NMDAR on the physiology of the kidney is not restricted to the glomerulus, it also modulates proximal tubular reabsorption (Slomowitz et al., 2004; Deng and Thomson, 2009) and is involved in the reno-renal reflex. In the renal pelvi, NR1 subunit expressed in afferent nerves, acts as a mechanoreceptor detecting increases in intrapelvic pressure. NMDAR activation increases afferent renal nerve activity and substance $\mathrm{P}$ release that induces diuresis and natriuresis. Moreover, intra-pelvic administration of D-serine mimicked NMDAR activation inducing renal sensory activation. In the renal pelvis SR is expressed in the muscle and in the uroepithelial layer suggesting a local effect of D-serine in physiological conditions (Ma et al., 2008).

In the corpus cavernosum of the penis, the vascular tone is essential for erection. In a flaccid state, the smooth muscles are tonically contracted allowing a small amount of arterial flow, an increase in the blood flow would produce erection. Here, penil neuronal NOS (PnNOS), NR1 and NR2 subunit of NMDAR are expressed in the cavernosa nerves where they are often colocalized (Magee et al., 2003). NMDARs activation induce a non-adrenergic non-cholinergic neurogenic relaxation of the corpus cavernosum reinforcing a tonic vasodilator effect of NMDAR on the vasculature (Gonzalez-Cadavid and Raifer, 2000; Ghasemi et al., 2010).

DAAO and SR are expressed in the corpus cavernosum and cavernosal membrane, respectively (Ghasemi et al., 2010; Kim 
et al., 2019) suggesting local regulation of NMDAR activity. In fact, D-serine administration induces a dose-dependent and NMDAR dependent relaxation of cavernosal tissue precontracted with phenylephrine. This effect was blocked by an Inhibitor of NOS (Ghasemi et al., 2010; Montesinos and Mani, 2016), although an inhibition of SR by NO has been shown in cultures of neurons (Watanabe et al., 2016), these results reinforce the interaction of $\mathrm{NO}$ and NMDAR in vasodilation. Further experiments are required to analyze the role of NMDAR and $\mathrm{D}$-serine in the erection in awake animals and points out possible therapeutic targets in the treatment of impotence.

Renal damage during aging is associated with podocytes dysfunction that can lead to matrix accumulation and glomeruloesclerosis that is manifested with a decrease in the blood flow, filtration rate, and an increase in the permeability causing proteinuria (Wiggins, 2012; Denic et al., 2016). All of this process depends on the proper function of NMDAR (Deng and Thomson, 2009; Giardino et al., 2009). In agreement with this, in mice kidneys homogenates there is an increase of calmodulin during aging, that acts as an inhibitor of NR1 subunit of NMDAR.

Aging is also associated with erectile dysfunction, a condition related with hypertension, decreased levels of testosterone, cardiovascular disease among many others. It has also been proposed that can be caused by a decrease in the effect of NO which is supported by a decrease in the NOS-containing nerve fibers in the penile of aged rats with erectile dysfunction (Gonzalez-Cadavid and Raifer, 2000). In this sense, Magee et al. (2003) did not find changes in the mRNA levels of PnNOS, nNOS and NMDAR in the penis of aged rats. However, further experiments are required to analyze the effect of aging on the levels of the proteins and the functionality of the NOS and NMDAR to rule out the involvement of NMDAR/NO expressed in cavernous nerves in erectile dysfunction. This is possible because cavernous nerves are required for the erectile responses mediated by hypothalamic medial preoptic area (MPOA) stimulation in adult male rats (Giuliano et al., 1997).

\section{ROLE OF NMDARS IN SKIN TURNOVER}

Skin is the first physical and anatomic barrier providing protection to the organism against environmental factors and agents such as UV radiation, heat, water loss, pathogens, etc. It is composed of three layers, the epidermis, the dermis, and the hypodermis. The epidermis is a self-renewing stratified and cornified epithelium divided into four layers: basal, spinous, granular, and the stratum corneum (Eckert et al., 2005). In the epidermis, keratinocytes are formed in the basal layer through cell division to replace terminally differentiated keratinocytes named corneocytes. The differentiation of the keratinocytes depends on the transglutaminases enzymes that catalyze covalent crosslinking of constituting proteins such as involucrin (IVL), loricrin (LRC), envoplakin (EVPL), periplakin (PPL), and small prolinerich proteins (SPRs) (Figure 2; Candi et al., 2005; Streubel et al., 2017). This calcium mediated crosslinking process contributes to the formation of the early cornified envelope (CE), that it is required to retain water and to limit the entry of the microbes and most chemicals through the skin (Kolarsick et al., 2011; Gilaberte et al., 2016).

NMDAR subunits are expressed in the stratum basale, spinosum, and granulosum of the epidermis (Fischer et al., 2004). Epidermal keratinocytes in the granular and cornified layers expressed SR localization (Inoue et al., 2014), suggesting an autocrine/paracrine effect of D-serine on the NMDAR expressed in the keratinocytes. Despite the detection of NMDAR subunits and SR in epidermal tissue, no activity of DAAO was detected (Jensen and Jacobsen, 1984). NMDRAs are involved in keratinocytes differentiation, proliferation (Fischer et al., 2004) and to maintain skin barrier and repair processes (Fuziwara et al., 2003). Cultured human keratinocytes treated with MK801 , showed a decrease in the proliferation and an increase in apoptosis (Fuziwara et al., 2003; Morhenn et al., 2004). In the same way, mice lacking serine racemase (SR-KO mice), showed significantly higher expression of filaggrin, involucrin, loricrin, and transglutaminase 3 in keratinocytes compared to control mice (Inoue et al., 2014). This suggests that a hypofunction of NMDAR would decrease the activity of the calcium dependent TGs leading to the accumulation of proteins necessary for the formation of the CE (Figure 2). In fact, in an assay of a barrier recovery, SR-KO mice had lower recovery rates after tape stripping than wild type mice revealing an important role of $\mathrm{D}$-serine in the healing process of the skin.

Skin aging is a complex biological process influenced by a combination of intrinsic and extrinsic factors (Cevenini et al., 2008). These factors lead together to structural and physiological alterations in the skin (Uitto, 1997; Shin et al., 2005). In particular, there is a decrease in the capacity of keratinocytes to proliferate (Yaar and Gilchrest, 2001), the epidermis thins and the rate of the turnover of the skin slows dramatically (Suter-Widmer and Elsner, 1996; Velarde et al., 2012). This contributes to a slow healing of minor injuries and weaker surgical scars. In this sense, tape stripping studies have revealed decreased cohesiveness in aged skin (Elias et al., 2002). In fact, barrier perturbation occurred after $18 \pm 2$ stripping in aged skin vs. $31 \pm 5$ stripping in control skin (Ghadially et al., 1995).

The alterations in the epidermis during aging (Yaar and Gilchrest, 1999) and the changes observed by interfering with NMDAR are very similar, suggesting a possible relationship between aging and a hypofunction for NMDAR. Furthermore, because SR and D-serine are required for the differentiation and maintenance of the physiological function of the skin (Inoue et al., 2014), it would be interesting to analyze the potential therapeutic effect of $\mathrm{D}$-serine to overcome the changes in keratinocytes differentiation caused by aging.

\section{ROLE OF D-SERINE IN THE HYPOFUNCTION OF NMDARS IN THE PERIPHERY}

Aging is a natural process that leads to reductions in maximal function and reserve capacity in all organ systems. Changes in the function of peripheral tissue due to aging result in 


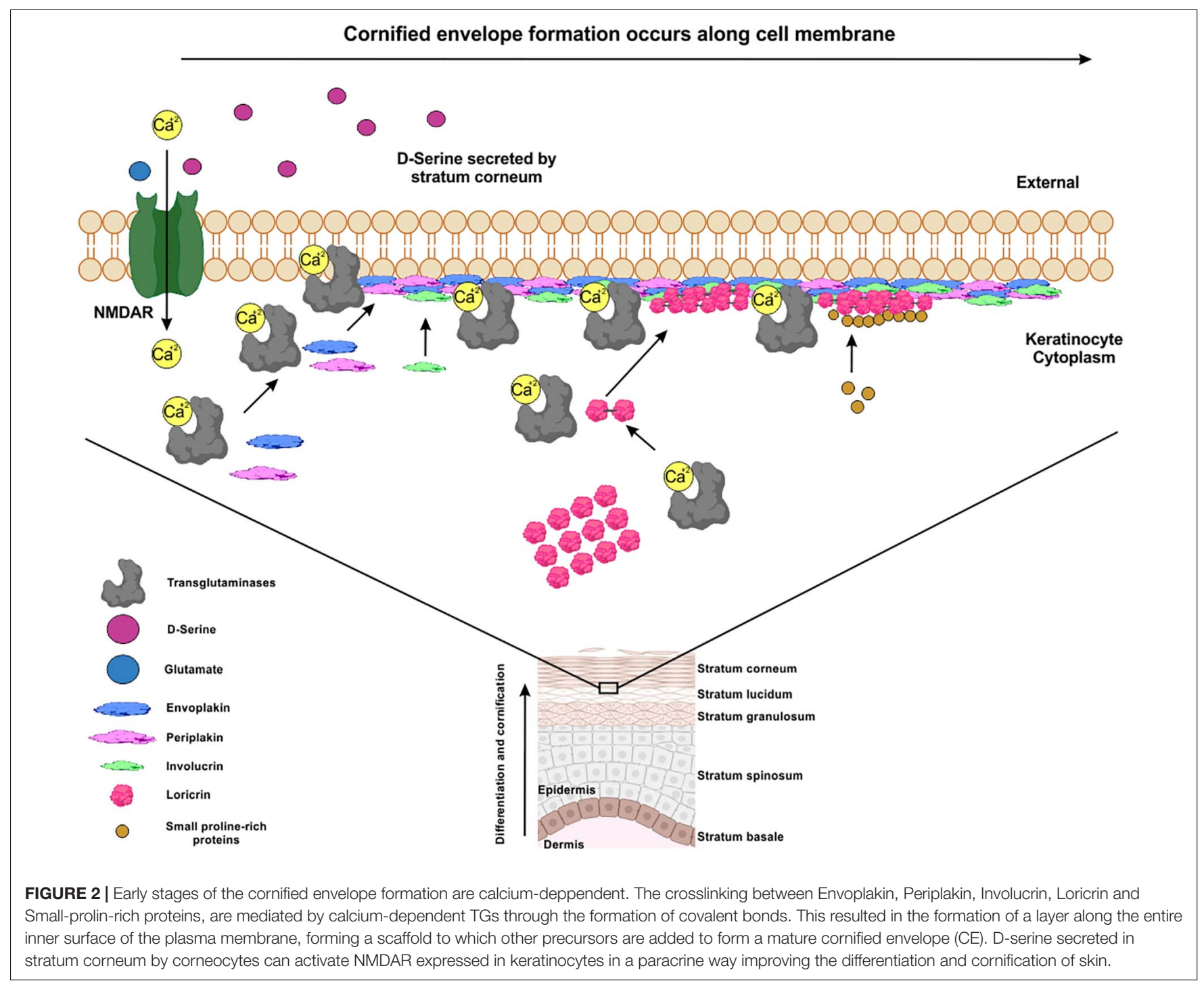

an increase of susceptibility to and frequency of disease, frailty, or disability. In fact, advancing age is the major risk factor for several chronic diseases in humans. Here, we provide evidence that the hypofunction of NMDARs resembles many manifestations observed during aging such as disturbed glomerular filtration, alterations in the skin turnover and bone remodeling, suggesting that NMDARs hypofunction could be associated with deterioration due to aging. We propose that a decrease in D-serine could account for this hypofunction because (1) NMDAR hypofunction in the brain associated with aging is related with a decrease in the synthesis of D-serine and not glycine (Mothet et al., 2006; Billard, 2018); (2) There is an increase of DAAO in the plasma associated with age (Lin et al., 2017); (3) NMDAR expressed in glomerular podocytes are insensitive to glycine (Anderson et al., 2011); (4) NMDAR activation induces vasodilation through the increase in NO production in a D-serine concentration-dependent manner (LeMaistre et al., 2012; Lu et al., 2019); (5) D-serine administration induces a dose-dependent and NMDAR dependent relaxation of cavernosal tissue pre-contracted with phenylephrine (Ghasemi et al., 2010); and (6) SR and NMDAR are localized in the keratinocytes of the skin (Inoue et al., 2014).

There are controversies regarding changes in plasma levels of D-serine associated with age that could account for a generalized hypofunction of NMDAR in the periphery. While some observed a negative association between D-serine levels and age (Avellar et al., 2016) others have observed an increase (Lin et al., 2017). However, plasma D-serine levels correlates with CSF or brain levels associated with malfunctioning of NMDAR in different pathologies like Schizophrenia and ALS (Hashimoto et al., 2003), and this does not occur with glycine plasma levels (Ohnuma et al., 2008; Lin et al., 2017). Although further experiments are required to analyze a decrease of D-serine in the blood with age, this evidence suggests that a decrease in D-serine levels in the brain could be reflected in blood levels. Furthermore, the quantification of local D-serine, SR and DAAO expression associated with aging 
in different tissues would shed light on the role of D-serine as the element regulating hypofunction on NMDAR in the periphery.

NMDAR containing NR1 subunit require both, the agonist and co-agonist, for the activation (Paoletti et al., 2013). This would limit an overactivation of NMDAR followed by $\mathrm{D}$-serine supplementation in one side, however, in the other side, if glutamate is also decrease in the periphery during aging it may not be D-serine sufficient to restore NMDAR hypofunction. D-Serine supplementation can cause necrosis of proximal straight tubules when administered at high doses (800 mg/kg) (Ganote et al., 1974), but it is well tolerated at low doses $(30 \mathrm{mg} / \mathrm{kg})$. This is important to take in consideration regarding a possible therapeutic supplementation of D-serine, in this sense it will be interesting to analyze the effect of low-doses in middle aged as a strategy to prevent a detriment with less risk of possible toxicity in the kidney.

D-serine can also be absorbed from the diet, especially from milk products (Brückner et al., 1992; Csapo et al., 2009) and synthesized by microorganisms of the intestinal microbiota, such as Firmicutes, Clostridia, Clostridiales, Lachnospiraceae, and Eisenbergiella (Matsumoto et al., 2018; Nakade et al., 2018). Supplementation with prebiotics such as fructo-oligosaccharides and galacto-oligosaccharides increased serum levels of D-serine and the expression of the NR1 and NR2A subunits in the prefrontal cortex (Savignac et al.,

\section{REFERENCES}

Abdelgawad, M. E., Delaisse, J. M., Hinge, M., Jensen, P. R., Alnaimi, R. W., and Rolighed, L. (2016). Early reversal cells in adult human bone remodeling: osteoblastic nature, catabolic functions and interactions with osteoclasts. Histochem. Cell Biol. 145, 603-615. doi: 10.1007/s00418-016-1414-y

Al-Suhaimi, E. A., and Al-Jafary, M. A. (2020). Endocrine roles of vitamin $\mathrm{K}$-dependent- osteocalcin in the relation between bone metabolism and metabolic disorders. Rev. Endocr. Metab. Disord. 21, 117-125. doi: 10.1007/ s11154-019-09517-9

Anderson, M., Suh, J. M., Young, E., and Stuart, E. (2011). Functional NMDA receptors with atypical properties are expressed in podocytes. Am. J. Physiol. Cell Physiol. 300, C22-C32.

Avellar, M., Scoriels, L., Madeira, C., Vargas-Lopes, C., Marques, P., and Dantas, C. (2016). The effect of D-serine administration on cognition and mood in older adults. Oncotarget 7, 11881-11888. doi: 10.18632/oncotarget.7691

Billard, J. M. (2018). Changes in serine racemase-dependent modulation of NMDA receptor: impact on physiological and pathological brain aging. Front. Mol. Biosci. 5:106.

Blantz, R. C., Deng, A., Lortie, M., Munger, K., Vallon, V., Gabbai, F. B., et al. (2002). The complex role of nitric oxide in the regulation of glomerular ultrafiltration. Kidney Int. 61, 782-785. doi: 10.1046/j.1523-1755.2002.00220.x

Brown, J. L., Kumbar, S. G., and Laurencin, C. T. (2013). Bone Tissue Engineering, in Biomaterials Science: an Introduction to Materials, 3rd Edn. Amsterdam: Elsevier.

Brückner, H., Jaek, P., Langer, M., and Godel, H. (1992). Liquid chromatographic determination of $\mathrm{D}$-amino acids in cheese and cow milk. Implication of starter cultures, amino acid racemases, and rumen microorganisms on formation, and nutritional considerations. Amino Acids 2, 271-284. doi: 10.1007/BF00805948

Candi, E., Oddi, S., Terrinoni, A., Paradisi, A., Ranalli, M., Finazzi-Agró, A., et al. (2001). Transglutaminase 5 cross-links loricrin, involucrin, and small proline-rich proteins in vitro. J. Biol. Chem. 276, 35014-35023. doi: 10.1074/ jbc.M010157200 doi: 10.1074/jbc.M010157200
2013). These findings raise the possibility of a restauration of D-serine levels with the diet to reduced NMDAR hypofunction. However, is not clear how intestinal D-serine transporters changes with aging, and would be important to determine the physiological and pathological levels of plasmatic D-serine.

\section{DATA AVAILABILITY STATEMENT}

The original contributions presented in the study are included in the article/supplementary material, further inquiries can be directed to the corresponding author/s.

\section{AUTHOR CONTRIBUTIONS}

ARV, ROM, and MLH edited the article. All authors drafted the article and approved the final version.

\section{FUNDING}

This work was funded by grants from CONACyT Ciencia de Frontera $171874(\mathrm{MLH})$, CONACyT Problemas Nacionales 2132 (MLH), PAPIIT-DGAPA IA208120 (MLH).

Candi, E., Schmidt, R., and Melino, G. (2005). The cornified envelope: a model of cell death in the skin. Nat. Rev. Mol. Cell Biol. 6, 328-340. doi: 10.1038/ nrm1619

Cevenini, E., Invidia, L., Lescai, F., Salvioli, S., Tieri, P., Castellani, G., et al. (2008). Human models of aging and longevity. Expert Opin. Biol. Ther. 8, 1393-1405.

Chan, A. W., Perry, S. G., Burch, H. B., Fagioli, S., Alvey, T. R., and Lowry, O. H. (1979). Distribution of two aminotransferases and D-amino acid oxidase within the nephron of young and adult rats. J. Histochem. Cytochem. 27, 751-755. doi: $10.1177 / 27.3 .39098$

Csapo, J., Albert, C., and Csapo-Kiss, Z. (2009). The D-amino acid content of foodstuffs (a review). Acta Univ. Sapient. Alim. 1, 5-30.

Deng, A., and Thomson, S. C. (2009). Renal NMDA receptors independently stimulate proximal reabsorption and glomerular filtration. Am. J. Physiol. 296, F976-F982.

Deng, A., Valdivielso, J. M., Munger, K. A., Blantz, R. C., and Thomson, S. C. (2002). Vasodilatory N-methyl-D-aspartate receptors are constitutively expressed in rat kidney. J. Am. Soc. Nephrol. 13, 1381-1384. doi: 10.1097/01.asn.0000013293. $11876.4 \mathrm{e}$

Denic, A., Glassock, R. J., and Rule, A. D. (2016). Structural and functional changes with the aging kidney. Adv. Chronic Kidney Dis. 23, 19-28. doi: 10.1053/j.ackd. 2015.08.004

Dobson, K. R., and Skerry, T. M. (2000). The NMDA-type glutamate receptor antagonist MK801 regulates differentiation of rat bone marrow osteoprogenitors and influences adipogenesis. J. Bone Miner. Res. 15(Suppl. 1), S72-S75.

Duffy, S. J., Tran, B. T., New, G., Tudball, R. N., Esler, M. D., and Harper, R. W. (1998). Continuous release of vasodilator prostanoids contributes to regulation of resting forearm blood flow in humans. Am. J. Physiol. 274, H1174-H1183.

Eble, J., and Niland, S. (2009). The extracellular matrix of blood vessels. Curr. Pharm. Des. 15, 1385-1400. doi: 10.2174/138161209787846757

Eckert, R. L., Sturniolo, M. T., Broome, A. M., Ruse, M., and Rorke, E. A. (2005). Transglutaminase function in epidermis. J. Invest. Dermatol. 124, 481-492. doi: 10.1111/j.0022-202x.2005.23627.x 
Ehlers, M. D., Zhang, S., Bernhadt, J. P., and Hunganir, R. L. (1996). Inactivation of NMDA receptors by direct interaction of calmodulin with the NR1 subunit. Cell 84, 745-755. doi: 10.1016/s0092-8674(00)81052-1

Elias, P. M., Ahn, S. K., Denda, M., Brown, B. E., Crumrine, D., Kimutai, L. K., et al. (2002). Modulations in epidermal calcium regulate the expression of differentiation-specific markers. J. Invest. Dermatol. 119, 1128-1136. doi: 10. 1046/j.1523-1747.2002.19512.x

Everts, V., Delaissé, J. M., Korper, W., Jansen, D. C., Tigchelaar-Gutter, W., Saftig, P., et al. (2002). The bone lining cell: its role in cleaning Howship's lacunae and initiating bone formation. J. Bone Miner. Res. 17, 77-90. doi: 10.1359/jbmr. 2002.17.1.77

Fischer, M., Glanz, D., William, T., Klapperstück, T., Wohlrab, J., and Marsch, W. C. H. (2004). N-methyl-D-aspartate receptors influence the intracellular calcium concentration of keratinocytes. Exp. Dermatol. 13, 512-519. doi: 10. 1111/j.0906-6705.2004.00200.x

Foltyn, V. N., Bendikov, I., De Miranda, J., Panizzutti, R., Dumin, E., Shleper, M., et al. (2005). Serine racemase modulates intracellular D-serine levels through an $\alpha, \beta$-elimination activity. J. Biol. Chem. 280, 1754-1763. doi: 10.1074/jbc. $\mathrm{m} 405726200$

Fuziwara, S., Inoue, K., and Denda, M. (2003). NMDA-type glutamate receptor is associated with cutaneous barrier homeostasis. J. Invest. Dermatol. 120, 1023-1029. doi: 10.1046/j.1523-1747.2003.12238.x

Ganote, C. E., Peterson, D. R., and Carone, F. A. (1974). The nature of D-serineinduced nephrotoxicity. Am. J. Pathol. 77, 269-282.

Genever, P. G., and Skerry, T. M. (2001). Regulation of spontaneous glutamate release activity in osteoblastic cells and its role in differentiation and survival: evidence for intrinsic glutamatergic signaling in bone. FASEB J. 15, 1586-1588. doi: 10.1096/fj.00-0594fje

Ghadially, R., Brown, B. E., Sequeira-Martin, S. M., Feingold, K. R., and Elias, P. M. (1995). The aged epidermal permeability barrier. structural, functional, and lipid biochemical abnormalities in humans and a senescent murine model. J. Clin. Invest. 95, 2281-2290. doi: 10.1172/jci117919

Ghasemi, M., Rezania, F., Lewin, J., Moore, K. P., and Mani, A. R. (2010). Dserine modulates neurogenic relaxation in rat corpus cavernosum. Biochem. Pharmacol. 79, 1791-1796. doi: 10.1016/j.bcp.2010.02.007

Giardino, L., Armelloni, S., Corbelli, A., Mattinzoli, D., Zennaro, C., and Guerrot, D. (2009). Podocyte glutamatergic signaling contributes to the function of the glomerular filtration barrier. J. Am. Soc. Nephrol. 20, 1929-1940. doi: 10.1681/ asn.2008121286

Gilaberte, Y., Prieto-Torres, L., Pastushenko, I., and Juarranz, Á (2016). Anatomy and function of the skin. Nanosci. Dermatol. 2016, 1-14. doi: 10.1002/ 9780470773093.ch1

Giuliano, F., Brown, B. K., Droupy, S., Benoit, G., and Rampin, O. (1997). Erectile response to hypothalamic stimulation in rats: role of peripheral nerves. Am. J. Physiol. 273, R1990-R1997.

Gonzalez-Cadavid, N. F., and Raifer, J. (2000). Therapeutic stimulation of penile nitric oxide synthase (NOS) and related pathways. Drugs Today 36, 163-174. doi: 10.1358/dot.2000.36.2-3.568790

Gradinaru, D., Borsa, C., Ionescu, C., and Prada, G. I. (2015). Oxidized LDL and NO synthesis - biomarkers of endothelial dysfunction and ageing. Mech. Ageing Dev. 151, 101-113. doi: 10.1016/j.mad.2015.03.003

Hashimoto, K., Fukushima, T., Shimizu, E., Komatsu, N., Watanabe, H., and Shinoda, N. (2003). Decreased serum levels of D-serine in patients with schizophrenia: evidence in support of the N-methyl-D-aspartate receptor hypofunction hypothesis of schizophrenia. Arch. Gen. Psychiatry 60, 572-576. doi: 10.1001/archpsyc.60.6.572

Hinoi, E., Fujimori, S., Takarada, T., Taniura, H., and Yoneda, Y. (2002). Facilitation of glutamate release by ionotropic glutamate receptors in osteoblasts. Biochem. Biophys. Res. Commun. 297, 452-458. doi: 10.1016/s0006$291 x(02) 02223-4$

Hinoi, E., Takarada, T., Uno, K., Inoue, M., Murafuji, Y., and Yoneda, Y. (2007). Glutamate suppresses osteoclastogenesis through the cystine/glutamate antiporter. Am. J. Pathol. 170, 1277-1290. doi: 10.2353/ajpath.2007.061039

Ho, M. L., Tsai, T. N., Chang, J. K., Shao, T. S., Jeng, Y. R., and Hsu, C. (2005). Down-regulation of $\mathrm{N}$-methyl D-aspartate receptor in rat-modeled disuse osteopenia. Osteoporos. Int. 16, 1780-1788. 10.1007/s00198-005$1928-y$
Inoue, R., Yoshihisa, Y., Tojo, Y., Okamura, C., Yoshida, Y., Kishimoto, J., et al. (2014). Localization of serine racemase and its role in the skin. J. Invest. Dermatol. 134, 1618-1626. doi: 10.1038/jid.2014.22

Itzstein, C., Cheynel, H., Burt-Pichat, B., Merle, B., Espinosa, L., Delmas, P. D., et al. (2001). Molecular identification of NMDA glutamate receptors expressed in bone cells. J. Cell. Biochem. 82, 134-144. doi: 10.1002/jcb.1114

Jensen, P. K., and Jacobsen, N. O. (1984). Studies of D-amino acid oxidase activity in human epidermis and cultured human epidermal cells. Arch. Dermatol. Res. 276, 57-64. doi: 10.1007/bf00412564

Jilka, R. L., Weinstein, R. S., Bellido, T., Parfitt, A. M., and Manolagas, S. C. (1998). Osteoblast programmed cell death (apoptosis): modulation by growth factors and cytokines. J. Bone Miner. Res. 13, 793-802. doi: 10.1359/jbmr.1998.13. 5.793

Kalinin, A. E., Kajava, A. V., and Steinert, P. M. (2002). Epithelial barrier function: assembly and structural features of the cornified cell envelope. BioEssays News Rev. Mol. Cell. Dev. Biol. 24, 789-800. doi: 10.1002/bies.10144 doi: 10.1002/ bies. 10144

Kim, S. H., Shishido, Y., Sogabe, H., Rachadech, W., Yorita, K., Kato, Y., et al. (2019). Age-and-gender-dependent D-amino acid oxidase activity in mouse brain and peripheral tissues: implication for aging and neurodegeneration. J. Biochem. 166, 187-196. doi: 10.1093/jb/mvz025

Knopf, F., Hammond, C., Chekuru, A., Kurth, T., Hans, S., Weber, C. W., et al. (2011). Bone regenerates via dedifferentiation of osteoblasts in the zebrafish fin. Dev. Cell 20, 713-724. doi: 10.1016/j.devcel.2011.04.014

Koibuchi, N., Matsuzaki, R. K., Ohtake, H., Niwa, A., and Yamaoka, S. (1995). Localization of D-amino acid oxidase mRNA in the mouse kidney and the effect of testosterone treatment. Histochem. Cell Biol. 104, 349-355. doi: 10. 1007/bf01458128

Kolarsick, P. A., Kolarsick, M. A., and Goodwin, C. (2011). Anatomy and Physiology of the Skin. J. Dermatol. Nurses Assoc. 3, 203-213.

Langdahl, B., Ferrari, S., and Dempster, D. W. (2016). Bone modeling, and remodeling: potential as therapeutic targets for the treatment of osteoporosis. Ther. Adv. Musculoskelet. Dis. 8, 225-235. doi: 10.1177/1759720x16670154

LeMaistre, J. L., Sanders, S. A., Stobart, M. J., Lu, L., Knox, J. D., Anderson, H. D., et al. (2012). Coactivation of NMDA receptors by glutamate and D-serine induces dilation of isolated middle cerebral arteries. J. Cereb. Blood Flow Metab. 32, 537-547. doi: 10.1038/jcbfm.2011.161

Lin, C. H., Yang, H. T., Chiu, C. C., and Lane, H. Y. (2017). Blood levels of D-amino acid oxidase vs. D-amino acids in reflecting cognitive aging. Sci. Rep. 7:14849.

Lu, L., Hogan-Cann, A. D., Globa, A. K., Lu, P., Nagy, J. I., Bamji, S. X., et al. (2019). Astrocytes drive cortical vasodilatoy signalin by activating endothelial NMDA receptors. J. Cereb. Blood Flow Metab. 39, 481-496. doi: 10.1177/ $0271678 \times 17734100$

Ma, M. C., Huang, H. S., Chen, Y. S., and Lee, S. H. (2008). Mechanosensitive $\mathrm{N}$-methyl-D-aspartate receptors contribute to sensory activation in the rat renal pelvis. Hypertension 52, 938-944. doi: 10.1161/hypertensionaha.108.114116

Magee, T. R., Ferrini, M. G., Davila, H. H., Zeller, C. B., Vernet, D., Sun, J., et al. (2003). Protein inhibitor of nitric oxide synthase (NOS) and the N-methyl$\mathrm{D}$-aspartate receptor are expressed in the rat and mouse penile nerves and colocalize with penile neuronal NOS. Biol. Reprod. 68, 478-488. doi: 10.1095/ biolreprod.102.007310

Massie, H. R., Aiello, V. R., and DeWolfe, L. K. (1989). Calcium and calmodulin changes with ageing in C57BL/6J mice. Gerontology 35, 100-105. doi: 10.1159/ 000213006

Matsumoto, C. S., Subramanian, S., and Fishbein, T. (2018). Adult intestinal transplantation. Gastroenterol. Clin. North Am. 47, 341-354.

Merle, B., Itzstein, C., Delmas, P. D., and Chenu, C. (2003). NMDA glutamate receptors are expressed by osteoclast precursors and involved in the regulation of osteoclastogenesis. J. Cell Biochem. 90, 424-436. doi: 10.1002/jcb.10625

Michel, T., and Vanhoutte. (2010). Cellular signaling and NO production. Pflugers Arch. Eur. J. Physiol. 459, 807-816.

Montesinos, C., and Mani, A. R. (2016). The role of D-serine in peripheral tissues. Eur. J. Pharmacol. 780, 216-223. doi: 10.1016/j.ejphar.2016.03.054

Morhenn, V. B., Murakami, M., O’Grady, T., Nordberg, J., and Gallo, R. L. (2004). Characterization of the expression and function of N-methyl-D-aspartate receptor in keratinocytes. Exp. Dermatol. 13, 505-511. doi: 10.1111/j.09066705.2004.00186.x 
Morimoto, R., Uehara, S., Yatsushiro, S., Juge, N., Hua, Z., Senoh, S., et al. (2006). Secretion of L-glutamate form osteoclasts through transcytosis. EMBO J. 25, 4175-4186. doi: 10.1038/sj.emboj.7601317

Mothet, J. P., Rouaud, E., Sinet, P. M., Potier, B., Jouvenceau, A., Dutar, P., et al. (2006). A critical role for the glia-derived neuromodulator D-serine in the agerelated deficits of cellular mechanisms of learning and memory. Aging Cell 5, 267-274. doi: 10.1111/j.1474-9726.2006.00216.x

Nakade, Y., Iwata, Y., Furuichi, K., Mita, M., Hamase, K., Konno, R., et al. (2018). Gut microbiota-derived D-serine protects against acute kidney injury. JCI Insight 3:e97957.

Nakamura, I., Gailit, J., and Sasaki, T. (1996). Osteoclast integrin $\alpha(v) \beta 3$ is present in the clear zone and contributes to cellular polarization. Cell Tissue Res. 286, 507-515. doi: 10.1007/s004410050720

Ohnuma, T., Sakai, Y., Maeshima, H., Hatano, T., Hanzawa, R., and Abe, S. (2008). Changes in plasma glycine, L-serine, and D-serine levels in patients with schizophrenia as their clinical symptoms improve: results from the Juntendo University Schizophrenia Projects (JUSP). Prog. Neuropsychopharmacol. Biol. Psychiatry 32, 1905-1912. doi: 10.1016/j.pnpbp.2008.07.022

Paoletti, P., Bellone, C., and Zhou, Q. (2013). NMDA receptor subunit diversity: impact on receptor properties, synaptic plasticity and disease. Nat. Rev. Neurosci. 14, 383-400. doi: 10.1038/nrn3504

Patton, A. J., Genever, P. G., Birch, M. A., Suva, L. J., and Skerry, T. M. (1998). Expression of an N-methyl-D-aspartate type receptor by human and rat osteoblasts and osteoclasts suggests a novel glutamate signaling pathway in bone. Bone 22, 645-649. doi: 10.1016/s8756-3282(98)00061-1

Ramage, L., Martel, M. A., Hardingham, G. E., and Salter, D. M. (2008). NMDA receptor expression and activity in osteoarthritic human articular chondrocytes. Osteoarthr. Cartil. 16, 1576-1584. doi: 10.1016/j.joca.2008.04.023

Sacchi, S., Rosini, E., Pollegioni, L., and Molla, G. (2013). D-amino acid oxidase inhibitors as a novel class of drugs for schizophrenia therapy. Curr. Pharm. Des. 19, 2499-2511. doi: 10.2174/1381612811319140002

Sandoo, A., Veldhuijzen, van Zanten, J., Metsios, G. S., Carroll, D., and Kitas, G. (2010). The endothelium and its role in regulating vascular tone. Open Cardiovasc. Med. J. 4, 302-312. doi: 10.2174/1874192401004010302

Sasabe, J., Suzuki, M., Miyoshi, Y., Tojo, Y., Okamura, C., Ito, S., et al. (2014). Ischemic acute kidney injury perturbs homeostasis of serine enantiomers in the body fluid in mice: early detection of renal dysfunction using the ratio of serine enantiomers. PLoS One 9:e86504. doi: 10.1371/journal.pone.008 6504

Savignac, H. M., Corona, G., Mills, H., Chen, L., Spencer, J. P., Tzortzis, G., et al. (2013). Prebiotic feeding elevates central brain derived neurotrophic factor, $\mathrm{N}$-methyl-D-aspartate receptor subunits and D-serine. Neurochem. Int. 63, 756-764. doi: 10.1016/j.neuint.2013.10.006

Sawa, N., Fujimoto, H., Sawa, Y., and Yamashita, J. (2019). Alternating differentiation and dedifferentiation between mature osteoblasts and osteocytes. Sci. Rep. 9:13842.

Steinert, P. M., and Marekov, L. N. (1999). Initiation of assembly of the cell envelope barrier structure of stratified squamous epithelia. Mol. Biol. Cell 10, 4247-4261. doi: 10.1091/mbc.10.12.4247 doi: 10.1091/mbc.10.12.4247

Shin, M. H., Rhie, G. E., Kim, Y. K., Park, C. H., Cho, K. H., Kim, K. H., et al. (2005). $\mathrm{H} 2 \mathrm{O} 2$ accumulation by catalase reduction changes MAP kinase signaling in Aged human skin in vivo. J. Invest. Dermatol. 125, 221-229. doi: 10.1111/j. 0022-202x.2005.23823.x

Slomowitz, L. A., Gabbai, F. B., Khang, S. J., Satriano, J., Thareau, S., Deng, A., et al. (2004). Protein intake regulates the vasodilatory function of the kidney and NMDA receptor expression. Renal Hemodynamics Cardiorenal Integration 287, R1184-R1189.

Streubel, M. K., Rinnerthaler, M., Bischof, J., and Richter, K. (2017). "Changes in the composition of the cornified envelope during skin aging: a calcium centric point of view," in Textbook of Aging Skin, eds M. Farage, K. Miller, and H. Maibach (Berlin: Springer).
Suter-Widmer, J., and Elsner, P. (1996). The Irritant Contact Dermatitis Syndrome. Baca Raton, FL: CRC Press.

Takarada, T., Takahata, Y., Iemata, M., Hinoi, E., Uno, K., Hirai, T., et al. (2009). Interference with cellular differentiation by $\mathrm{D}$-serine through antagonism at N-Methyl-D-Aspartate receptors composed of NR1 and NR3A subunits in chondrocytes. J. Cell Physiol. 220, 756-764. doi: 10.1002/jcp.21821

Takarada, T., Takarada-Iemata, M., Takahata, Y., Yamada, D., Yamamoto, T., Nakamura, Y., et al. (2012). Osteoclastogenesis is negatively regulated by D-serine produced by osteoblasts. J. Cell Physiol. 227, 3477-3487. doi: 10.1002/ jcp. 24048

Tennant, M., and McGeachie, J. K. (1990). Blood vessel structure and function: a brief update on recent advances. Aust. N. Z. J. Surg. 60, 747-753. doi: 10.1111/ j.1445-2197.1990.tb07468.x

Touaitahuata, H., Cres, G., de Rossi, S., Vives, V., and Blangy, A. (2014). The mineral dissolution function of osteoclasts is dispensable for hypertrophic cartilage degradation during long bone development and growth. Dev. Biol. 393, 57-70. doi: 10.1016/j.ydbio.2014.06.020

Uitto, J. (1997). Understanding premature skin aging. N. Engl. J. Med. 337, 1463-1465. doi: 10.1056/nejm199711133372011

Velarde, M. C., Flynn, J. M., Day, N. U., Melov, S., and Campisi. (2012). Mitochondrial oxidative stress caused by Sod2 deficiency promotes cellular senescence and aging phenotypes in the skin. Aging 4, 3-12. doi: 10.18632/ aging. 100423

Watanabe, A., Sasaki, T., Yukami, T., Kanki, H., Sakaguchi, M., Takemori, H., et al. (2016). Serine racemase inhibition induces nitric oxide-mediated neurovascular protection during cerebral ischemia. Neuroscience 339, 139-149. doi: 10.1016/ j.neuroscience.2016.09.036

Wiggins, J. (2012). Aging in the glomerulus. J. Gerontol. A Biol. Sci. Med. Sci. 67, 1358-1364.

Wolosker, H., Sheth, K. N., Takahashi, M., Mothet, J. P., Brady, R. O., Ferris, C. D., et al. (1999). Purification of serine racemase: biosynthesis of the neuromodulator D-serine. Proc. Natl. Acad. Sci. USA 96, 721-725. doi: 10.1073/ pnas.96.2.721

Wu, M., Chen, G., and Li, Y. P. (2016). TGF- $\beta$ and BMP signaling in osteoblast, skeletal development, and bone formation, homeostasis and disease. Bone Res. 4:16009.

Wu, Q., Zhou, X., Huang, D., Ji, Y., and Kang, F. (2017). IL-6 Enhances osteocytemediated osteoclastogenesis by promoting JAK2 and RANKL activity in vitro. Cell. Physiol. Biochem. 41, 1360-1369. doi: 10.1159/000465455

Xia, M., Liu, Y., Figueroa, D. J., Chiu, C. S., Wei, N., Lawlor, A. M., et al. (2004). Characterization and localization of a human serine racemase. Brain Res. Mol. Brain Res. 125, 96-104.

Yaar, M., and Gilchrest, B. A. (1999). “Aging of skin,” in Fitzpatrick's Dermatology in General Medicine, 5th Edn, Vol. 1, eds I. M. Freedberg, A. Z. Eisen, and K. Wolff (New York: McGraw-Hill).

Yaar, M., and Gilchrest, B. A. (2001). Ageing and photoageing of keratinocytes and melanocytes. Clin. Exp. Dermatol. 26, 583-591. doi: 10.1046/j.1365-2230.2001. 00895.X

Yoneda, Y. (2017). NMDA Receptor in Bone. Totowa, NJ: Humana Press Inc.

Conflict of Interest: The authors declare that the research was conducted in the absence of any commercial or financial relationships that could be construed as a potential conflict of interest.

Copyright (c) 2021 Rivera-Villaseñor, Higinio-Rodríguez, Nava-Gómez, VázquezPrieto, Calero-Vargas, Olivares-Moreno and López-Hidalgo. This is an open-access article distributed under the terms of the Creative Commons Attribution License (CC BY). The use, distribution or reproduction in other forums is permitted, provided the original author(s) and the copyright owner(s) are credited and that the original publication in this journal is cited, in accordance with accepted academic practice. No use, distribution or reproduction is permitted which does not comply with these terms. 